\title{
Review
}

\section{Telemedicine in the management of patients with chronic respiratory failure}

Patients with chronic respiratory failure are often required to attend multiple hospital appointments, which may be difficult due to their physical disabilities and the amount of equipment they are required to bring. Their caregivers often struggle with the lack of immediate care available when the patient suffers difficulties at home. Telemedicine is an opportunity to bridge the gap between home and healthcare professionals by allowing the healthcare team to reach into patients' homes to provide more frequent support. The evidence for the use of telemedicine in patients with chronic respiratory failure remains equivocal. Although the uptake of telemedicine has been slow, the SARSCoV-2 pandemic has resulted in the rapid dissemination of telemedicine to allow the delivery of care to vulnerable patients while reducing the need for their attendance in hospital. Logistical and legal challenges to the delivery of telemedicine remain, but the pandemic may serve as a driver to ameliorate these challenges and facilitate wider use of this technology to improve the experience of patients with chronic respiratory failure.

\section{Educational aims}

- To provide an overview of the rationale for delivering care via telemedicine for patients with chronic respiratory failure.

- To provide the evidence base for establishing a telemedicine service.

- To highlight the potential opportunities and challenges in delivering a telemedicine service for patients with chronic respiratory failure.
Telemedicine is the remote diagnosis and management of patients by telecommunications technology. Prior to the severe acute respiratory syndrome coronavirus 2 (SARS-CoV-2) pandemic this treatment modality was slowly being adopted, but faced challenges around the availability of technology, acceptance of the technology by patients and healthcare professionals, legal issues
@ERSpublications

The SARS-CoV-2 pandemic has sped up the adoption of telemedicine for chronic respiratory failure. The opportunity to make significant progress towards its widespread adoption should now be taken to improve the delivery of care for these patients. https://bit.ly/3qSu7d1
Cite as: Shah NM, Kaltsakas G. Telemedicine in the management of patients with chronic respiratory failure. Breathe 2021; 17 : 210008. 
around the transmission of confidential data and financing of a telemedicine system [1]. An umbrella review of countries within the Organisation for Economic Co-operation and Development (OECD) demonstrated the varied reports of effectiveness of telemedicine and barriers to its uptake. An important barrier was the low-quality evidence available in this field [2, 3], particularly for respiratory conditions $[4,5]$. The impact on the delivery of conventional healthcare precipitated by the SARS-CoV- 2 pandemic has provided an impetus to health systems worldwide to hasten the adoption of remote and telemedicine [6]. This review assesses the value of telemedicine in the management of patients with chronic respiratory failure, how it is currently used and explores opportunities for the future. Keywords searched for included "telemedicine", "telehealth", "telerehabilitation" and "respiratory failure". A systematic review of the literature was not performed.

In this review, the term "chronic respiratory failure" encompasses both chronic hypoxic and hypercapnic respiratory failure. Chronic hypoxic respiratory failure can be caused by conditions such as COPD, interstitial lung disease, bronchiectasis and cystic fibrosis (CF), amongst many others. To treat chronic hypoxia in these conditions patients often receive long-term oxygen therapy (LTOT) at home [7]. Chronic hypercapnic respiratory failure can be caused by any condition contributing to a ventilation defect, such as COPD, neuromuscular diseases, chest wall diseases and obesity hypoventilation syndrome. There is an increasing body of evidence that suggests patients with chronic hypercapnic failure should be treated with domiciliary home mechanical ventilation (HMV) $[8,9]$. Patients with both forms of respiratory failure require frequent monitoring by both primary and secondary care, and suffer frequent emergency department visits and hospitalisations $[10,11]$.

\section{Rationale for telemedicine in chronic respiratory failure}

Chronic respiratory failure can have a significant impact on the health-related quality of life (QoL) of patients. These patients require a multidisciplinary management approach, which can often result in numerous appointments with different specialists, all necessitating visits to hospital [12]. This can present difficulties for the patient, especially as many suffer from limited mobility due to comorbidities and the presence of extensive equipment [13]. Although there is now strong evidence for the use of HMV in patients with chronic hypercapnic respiratory failure for its effects on mortality and reducing hospital admissions [14], this effect is less clear for LTOT [15]. Therefore, it is important that these treatments do not have a negative impact on QoL. Investigations of the impact on QoL are mixed. Some studies suggest these treatment modalities improve QoL [16-18], while others report no change [19-22]. Perhaps unsurprisingly, patients receiving domiciliary invasive mechanical ventilation reported significantly impaired QoL $[23,24]$. The specific effect of the presence of devices in the home and the impact of multiple hospital visits on QoL have not been extensively investigated [25]. The impact on caregiver QoL must also be considered. An early study reported significant frustrations from caregivers about being able to manage challenges quickly and effectively, due to lack of education about the treatment [26]. Qualitative data about the experiences of patients and caregivers on the transition to HMV also demonstrated that frustrations arise from the lack of knowledge and ability to obtain help efficiently [27]. Although not extensively investigated, one can surmise that patients and caregivers may benefit from the opportunity for more frequent input into their care, and that a reduction in the number of hospital visits is likely to provide benefit to their health-related QoL. A European Respiratory Society statement emphasised the need to reduce the burden of care on caregivers for patients receiving $\mathrm{HMV}$ by increasing the use of telemonitoring, while also illustrating the challenges that exist preventing this [28].

A feature of the traditional management of this patient group is a mobile home service, which visits patients in their home and can be an effective method to provide more regular care to patients who find it difficult to attend in-hospital visits and reduce the burden of care on caregivers. Services they may provide include clinical review, ventilator servicing, education and training, and tracheostomy change for patients receiving invasive mechanical ventilation. A recent qualitative study demonstrated the support a home care team provides to patients receiving long-term invasive mechanical ventilation [29]. Although clearly beneficial, this service presents a logistical and financial burden that must be considered. A telemedicine service could help to reduce this burden by potentially reducing the frequency of in-person review of the patients. Furthermore, during the SARS-CoV-2 pandemic, direct interaction with these vulnerable patients has been limited [30]. Telemedicine is likely to be a useful means of maintaining contact and providing support to patients, without the need for physical appointments.

\section{Use of telehealth in chronic respiratory failure}

The delivery of telemedicine can include direct consultations with the patient using remote technology, as well as indirect interactions such as the wireless delivery of data from the patient to the clinical team, wireless manipulation of treatment by the clinician, and monitoring of patient parameters using wearable sensors and associated smartphone applications [31]. This may 


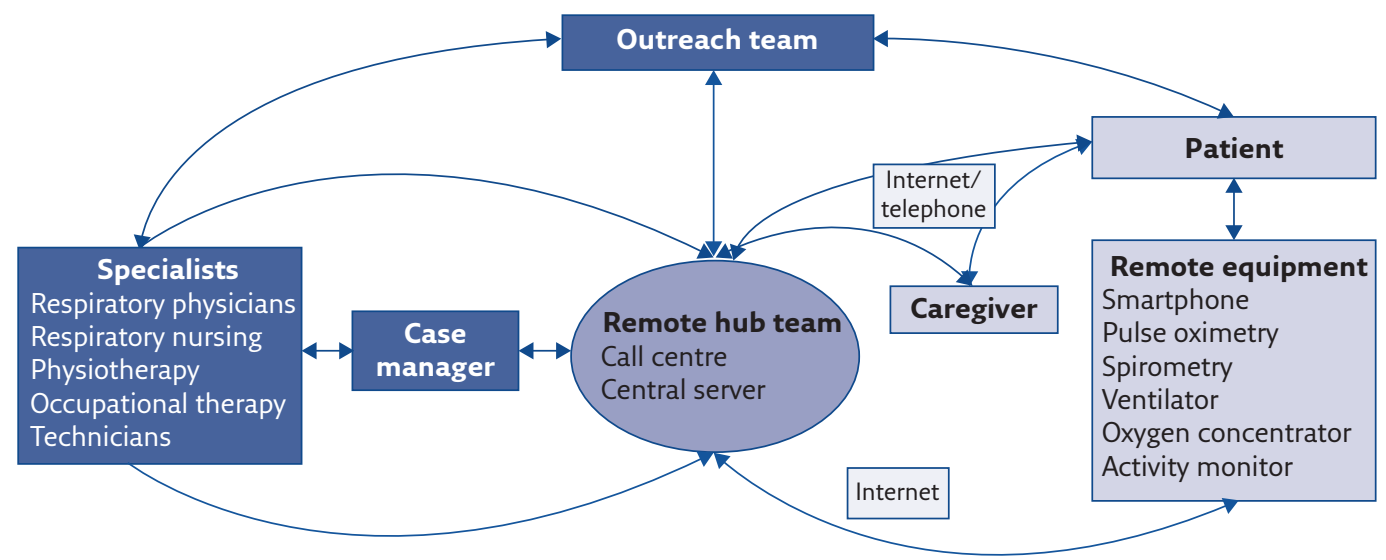

Figure 1 Schematic demonstration of a proposed model for a telemedicine service for patients with chronic respiratory failure. The patient can contact their healthcare provider through a central call centre. All remote data will be transmitted through a central server. Information collected at the remote hub can then be transmitted to the specialist teams via a personal case manager. The specialist teams can then communicate back to the patient and/or the equipment via the remote hub, or through outreach services who can then visit the patient. This model is likely to evolve as newer technologies become available. This schematic of a proposed telemedicine service has been designed based upon previously published descriptions [12, 124-126].

result in a reduced need for conducting in-person consultations and overnight in-hospital studies that increase the burden on the patient. More frequent and closer monitoring of these patients may also reduce the incidence of exacerbations and the need for hospitalisation. AliverTI [32] comprehensively reviewed the potential role of wearable devices in patients with a broad spectrum of respiratory conditions, including the monitoring of pulse oximetry, minute ventilation and pattern of breathing, physical activity and local air quality. For the delivery of pulmonary telerehabilitation, a systematic review identified that remote technologies can be used to define exercise plans, generate activity reports, provide patient education and schedule exercise tasks [33]. The exact set-up of a telemedicine service will vary depending upon the local healthcare system and the availability of infrastructure and resources; in figure 1 we propose a suggested model for a telemedicine service for patients with chronic respiratory failure that can be adapted to fit local requirements. While we have incorporated a central call centre and server (the "remote hub team"), it is recognised that not all healthcare systems will have the ability to resource this. Different configurations of our proposed model, such as direct transmission from the patient to the specialist team with/without a case manager, would also be plausible. There is a wide scope for the adoption of telemedicine practices to augment care specifically in patients with chronic respiratory failure; the data to support this is of varied quality and is reviewed in the following sections (table 1).

\section{Hypoxic respiratory failure}

\section{COPD}

The use of telemedicine has been investigated most in patients with COPD. Surveys of COPD practitioners have demonstrated that approximately one third are currently using telemedicine approaches in their practice [34, 35]. In a cohort of patients with primarily severe COPD (Global Initiative for Chronic Obstructive Lung Disease (GOLD) grade IV, group $D$ patients), the rate of hospitalisation was reduced compared with the expected values after adoption of a telemonitoring system, without any increase in healthcare cost [36]. Similarly, a cluster randomised study investigated the addition of a telehealth programme, which involved the daily remote assessment of heart rate, pulse oximetry and blood pressure and assessment of peak expiratory flow three times per week, to the care of patients with COPD receiving LTOT. After 7 months of follow-up, individuals enrolled into the telehealth programme reported fewer emergency department visits, hospital admissions, reduced hospital length of stay and requirement for NIV [37]. A randomised controlled trial compared usual care with a tele-assistance programme (which involved wearing a pulse oximeter that transmitted peripheral oxygen saturation to the clinical team via a home modem) in patients with either LTOT and/or HMV [38]. This nurse-centred programme resulted in fewer hospitalisations and primary care interactions, with no increased cost burden. This effect was observed most in the COPD cohort. Conversely, a large randomised multicentre study comparing remote monitoring of forced oscillation technique with usual care failed to demonstrate any benefit of closer and more frequent review of airway mechanics on time to hospitalisation or QoL [39]. A small cohort of patients receiving LTOT underwent QoL assessment (36-item Short-Form Health Survey (SF-36)) before and 3 months after the initiation of a telemonitoring programme. The telemonitoring included remote monitoring use of the oxygen concentrator as well as pulse oximetry. Although summary SF-36 score did not improve, 
Table 1 Potential benefits of telemedicine-delivered care in patients with conditions causing chronic respiratory failure

\begin{tabular}{|c|c|}
\hline Condition & Benefit \\
\hline COPD & $\begin{array}{l}\text { Reduction in exacerbations, emergency department visits } \\
\text { and hospitalisations }[36-38,63,79] \\
\text { Improved QoL [40] } \\
\text { Home set-up of noninvasive ventilation (NIV) }[76,92]\end{array}$ \\
\hline CF & $\begin{array}{l}\text { Reduced time spent attending appointments [44] } \\
\text { Increased frequency of clinical review [49] } \\
\text { Financial savings [46] }\end{array}$ \\
\hline Non-CF bronchiectasis & None identified \\
\hline Idiopathic pulmonary fibrosis & Improved psychological well-being [56] \\
\hline Amyotrophic lateral sclerosis (ALS) & $\begin{array}{l}\text { Reduction in hospital presentations and admissions [83] } \\
\text { Reduced in-person clinical encounters [82] } \\
\text { Financial savings [86-88] }\end{array}$ \\
\hline $\begin{array}{l}\text { Neuromuscular conditions and } \\
\text { chest wall diseases }\end{array}$ & $\begin{array}{l}\text { Reduction in emergency presentations [94, 98, 101, 102] } \\
\text { Reduced in-person clinical reviews [99-101] } \\
\text { Home set-up of NIV [93] } \\
\text { Domiciliary-based chest physiotherapy [94] } \\
\text { Financial savings [93] }\end{array}$ \\
\hline Obesity hypoventilation syndrome & None identified \\
\hline
\end{tabular}

the vitality and mental health sub-scores did improve significantly following the initiation of the programme, suggesting telemonitoring may have a role in the maintenance of QoL in these patients [40]. Another approach using remote monitoring of oximetry and activity using an accelerometer in patients requiring LTOT was well received, with good patient satisfaction [41]. The combination of oximetry and activity may allow the remote titration of oxygen prescription based upon how the patient's daily activities change. Finally, a telemedicine programme delivering palliative care consultations remotely to patients with severe COPD to discuss advanced care plans was feasible and well accepted in the small sample population of 10 patients [42]. Although a small study, this study is a useful proof-of-concept for healthcare systems where palliative care manpower may be limited or patients are geographically dispersed, making home visits challenging. Despite these mostly encouraging findings, a recent metaanalysis comparing telemonitoring with usual care in COPD reported that telemonitoring was unlikely to provide significant benefit on health outcomes [43]. It should be noted that this meta-analysis included studies investigating patients with COPD of any severity; an adequate number of randomised studies in patients with advanced COPD have not yet been conducted to warrant meta-analysis.

\section{Cystic fibrosis}

Patients with CF are frequently seen in outpatient services for monitoring and treatment, necessitating frequent visits to hospital. Telemedicine has been investigated for its potential to reduce this clinic burden for these patients. Remote monitoring of spirometry is feasible. In Australian children with CF it saved $\sim 5 \mathrm{~h}$ travel time per visit [44] with an obvious benefit on school attendance, which is a priority for school-age CF patients [45]. A modelling study based on data from 4 years of follow-up demonstrated that remote spirometry could result in financial savings of EUR 35000-40000 per patient over 10 years [46]. Patient satisfaction from daily remote monitoring was high [47] suggesting that the implementation of such a programme is feasible.

The impact of telemedicine on CF patient outcomes is less clear. Programmes to provide on-demand appointments via videoconferencing [48] and remote daily monitoring of spirometry and oxygen saturation with twice-weekly review [49] resulted in an increase in the number of clinics per patient and health-related QoL, with high patient satisfaction. These programmes also resulted in an increase in the number of antibiotic courses prescribed. Although this could be interpreted as a negative impact, it may also reflect that closer monitoring of these patients resulted in more infections being detected. A case-control study comparing standard care with a home telemonitoring system demonstrated that patients in the telemonitoring arm suffered significantly fewer infections with Pseudomonas aeruginosa than those in the usual care arm. In addition, there was a less prominent decline in forced expiratory volume in $1 \mathrm{~s}\left(\mathrm{FEV}_{1}\right)$ in the telemonitoring arm [50]. Conversely, a randomised controlled trial comparing weekly telemedicine assessment with 
usual care in patients awaiting lung transplant failed to demonstrate any difference in number of primary and secondary care visits, use of antibiotics or hospitalisations [51]. As a result of these data, reviews of the evidence have been unable to make a strong case for the implementation of telemedicine into CF programmes [52, 53], but given its potential to reduce the burden on patients there is value in conducting larger prospective randomised studies to identify any benefits. Despite the lack of clear evidence, the SARS-CoV-2 pandemic has forced the introduction of telemedicine services in CF to facilitate continued patient management and has been met with little resistance from either patients or healthcare professionals [54]. Interestingly, to the best of our knowledge, telemedicine has not been investigated in patients with non-CF bronchiectasis.

\section{Interstitial lung disease}

The uptake of telemedicine in interstitial lung diseases has been limited. A proof-of-concept study investigated the feasibility of using daily remote spirometry in patients with idiopathic pulmonary fibrosis [55]. This study demonstrated good agreement between in-person and remote spirometry and that patients found it a useful intervention in their management. The same group has recently published a randomised controlled study in idiopathic pulmonary fibrosis comparing usual care with a home monitoring package (remote spirometry, symptom reporting, patientreported outcomes, medication coaching and electronic consultations). There was no significant difference in QoL between the two groups, but the psychological domain of the King's Brief Interstitial Lung Disease Questionnaire was higher in the home monitoring group [56]. A pilot study investigating the value of supervised remote video gaming to deliver pulmonary rehabilitation in patients with idiopathic pulmonary fibrosis failed to demonstrate any benefit in exercise capacity, breathlessness or QoL after a 12-week intervention [57]. The SARSCoV-2 pandemic has resulted in a temporary increase in the use of telemedicine in this patient group [58]. While designing studies in this patient group, who can have rapidly progressive disease, can be difficult, if well-designed studies with targeted interventions can demonstrate noninferiority or superiority compared with standard care, they are likely to act as catalysts for an increase in the use of telemedicine.

\section{Pulmonary rehabilitation}

Patients with respiratory failure are often referred for pulmonary rehabilitation to help maintain respiratory health and reduce physical decline. Access to this service can be variable due to provision of appropriately trained professionals and the system of renumeration. Telemedicine has been investigated as a method to widen access to a larger group of patients. Large-scale videoassisted pulmonary rehabilitation programmes have already been introduced in the USA [59] and Canada [60], demonstrating feasibility and patient acceptance. An international survey of pulmonary rehabilitation practitioners revealed that $80 \%$ had a positive intention to use telerehabilitation [61]. Indeed, in response to the SARS-CoV-2 pandemic, an Australian case series demonstrated that the pulmonary rehabilitation practitioners were already well-versed and prepared to deliver a telemedicinebased programme with success [62]. It is important to determine whether telerehabilitation has a positive impact on patient outcomes.

A randomised study compared telemedicinebased maintenance rehabilitation with hospitalbased outpatient maintenance rehabilitation following a pulmonary rehabilitation programme for COPD patients. They demonstrated that the telemedicine-based programme was equally effective in reducing exacerbations of COPD and hospitalisations, and was more effective in reducing emergency department visits [63]. An observational study in COPD patients demonstrated that the delivery of a video-based rehabilitation programme following an exacerbation reduced the 30-day readmission rate compared with usual care [64]. Conversely, a multicentre randomised study comparing telerehabilitation with outpatient-based pulmonary rehabilitation did not demonstrate any significant difference in exercise capacity, hospitalisation rate or mortality at 12 months [65]. Similarly, another multicentre randomised study failed to demonstrate any benefit of telerehabilitation over usual care on exercise tolerance, symptomatology or healthrelated QoL [66].

Technical challenges with delivering telerehabilitation have also been reported. Smartphone-based oximetry is not a reliable measure of oxygen saturation during exercise [67], and so this may impact on safety during remotely monitored exercise programmes. In addition, smartphone use during a 12-week remotely monitored pulmonary rehabilitation programme fell significantly with time, suggesting that the current technology available may not be suitable for the target population to maintain engagement over an extended period of time [68]. Other issues of concern that persist in the delivery of telerehabilitation include the risk of falls in this elderly population conducting independent exercise, the inability to rehearse correct technique with a practitioner and the lack of suitable equipment in the home. Further work is required to design appropriate and safe programmes that can be delivered remotely, without increasing the risk of falls and injury to the patient. Currently, without the physical presence of a practitioner, the level of difficulty of any telerehabilitation programme is limited [59].

A qualitative investigation about the beliefs of patients on the place of telemedicine-assisted 
rehabilitation demonstrated that, while patients are not averse to it, several issues need to be considered when implementing a programme [69]. Most patients attending pulmonary rehabilitation are older; concern was raised about the familiarity of older people with the technology required and that a period of education about the devices used may be necessary. Patients commented on the benefit they receive from the social interaction with other patients, and their concern at this loss if using remote programmes. Positively, they also noted that the ability to have wearable devices at home that provide them with immediate feedback about their activity can be empowering.

A systematic review of telerehabilitation in COPD concluded that while it provides benefit in terms of exercise capacity and QoL over no intervention, these benefits are similar to those delivered via traditional inpatient or outpatient programmes [70]. A recent Cochrane review on telerehabilitation for chronic respiratory disease concluded that while outcomes delivered by telemedicine are similar to traditional pulmonary rehabilitation programmes, the certainty of this conclusion is limited by the dearth of highquality evidence available. Furthermore, they noted that studies need to be conducted in diseases other than COPD, on the economic efficacy of telerehabilitation and the duration of benefit beyond the main intervention, to allow more conclusive assessments to be made [71]. A potential trigger for these studies to be conducted is the onset of the SARS-CoV-2 pandemic. The pandemic has resulted in most in-person pulmonary rehabilitation programmes stopping, with the consequent potential clinical impact on this vulnerable population [72]. The need for the formalisation of telerehabilitation in respiratory failure with the development of a strong evidence base and guidelines to inform programme development has been acknowledged and may instigate more studies being conducted in this field [73].

\section{Hypercapnic respiratory failure}

For patients receiving HMV for hypercapnic respiratory failure, telemonitoring can be delivered through a cloud-based system, which allows transmission of vital signs from the patient and data about usage patterns, adherence and physiology from the ventilator. This also allows the healthcare team to adjust the ventilator settings based on the information received. Telemedicine has been used while setting patients up on HMV, as well as to monitor and titrate their treatment. A survey of HMV users reported that approximately half of HMV users and $60 \%$ of their carers would welcome telemonitoring [74]. An international survey of physicians delivering domiciliary NIV conducted in 2013 reported that only 5\% offered a telehealth programme for monitoring COPD patients [75].

\section{COPD}

A recent randomised study demonstrated that the initiation of NIV at home with the assistance of telemedicine, in patients with COPD, was noninferior to the initiation of NIV in hospital [76]. The primary outcome assessed was change in daytime arterial carbon dioxide tension $\left(\mathrm{PaCO}_{2}\right)$ after 6 months of NIV. An early feasibility study demonstrated that a telemonitoring system in patients requiring HMV, involving once a week nocturnal monitoring and the provision of 24/7 telephone counselling, was feasible and acceptable to patients [77]. Remote monitoring of ventilator parameters (e.g. respiratory rate and the percentage of respiratory cycles triggered by the patient) was predictive of exacerbations as assessed by the EXACT-Pro questionnaire [78]. A retrospective study reported on the impact of the addition of teleassistance to COPD patients receiving LTOT with or without NIV. The tele-assistance programme involved continuous monitoring with pulse oximetry with 24-h availability of a pulmonologist or respiratory nurse to provide advice. The addition of tele-assistance reduced the risk of hospitalisation in the LTOT cohort and the risk of exacerbation in the NIV cohort [79]. Conversely, a randomised controlled study comparing usual care with an extensive telemonitoring system in patients with chronic respiratory failure who had suffered from an exacerbation reported no difference in time to the subsequent admission or QoL. In fact, the study reported an increase in hospitalisation rate in the telemonitoring arm [80].

\section{Amyotrophic lateral sclerosis}

There is understandable interest in the value of telemedicine in ALS, due to the significantly debilitating and rapidly progressive nature of this condition. The addition of a modem to a ventilator, to transmit ventilator parameters to the clinical team and to allow the clinical team to adjust ventilator settings has been demonstrated to be feasible, safe and well accepted by patients [81]. A 15-year retrospective report on the activity of the Maugeri Centre for Telehealth and Telecare demonstrated that a nurse tutor remotely delivered $50 \%$ of interactions for patients with ALS, which reduced the need for review by the pulmonologist and for in-hospital review [82]. Similarly, a prospective single-blind study demonstrated that the addition of a modem to the ventilator resulted in significantly lower emergency presentations and hospitalisations. However, the telemedicine arm did experience an increase in the need for intervention during the initial establishment of NIV, and this effect should not be discounted as the burden on the healthcare team can substantially increase as a result [83]. A retrospective cohort study compared ALS patients who chose to receive telemedicine against those who chose usual care. There was no difference 
in survival time between the groups, and the telemedicine cohort had a lower rate of decline, although this may be explained by a better baseline state directing their choice to receive telemedicine [84]. A qualitative study in ALS patients revealed that telemedicine is well received. Patients found this approach empowering in promoting their well-being and they welcomed the potential reduction in clinical contact [85]. Telemonitoring of the NIV device resulted in cost savings of EUR 700 per patient per year, compared with usual care [86]. Similarly, a telemedicine service for ALS resulted in financial savings compared with usual care in the USA [87] and the provision of on-demand mechanical insufflation-exsufflation (MI-E) following remote consultations, compared with long-term loans of the $\mathrm{MI}-\mathrm{E}$ device resulted in $59 \%$ less expense [88]. Although cost-effectiveness will depend on the local healthcare renumeration system, it is likely that by reducing the number of physical patient consultations, and possibly hospitalisations, the per-patient cost of care will improve. Despite these supportive studies, a systematic review has demonstrated that a key barrier to the use of telemedicine in ALS is healthcare professional reticence, due to perceived legal and financial challenges that have not yet been addressed [89]. Another review of the literature also highlighted the lack of studies and understanding of physician legal responsibilities and financing as an outstanding challenge to the delivery of telemedicine in ALS [90]. Finally, a systematic review investigating the use of telehealth specifically to improve access to care for ALS reported on various methods identified in the literature but that the quality of evidence was too low to provide firm conclusions [91].

\section{Other neuromuscular conditions}

Patients with neuromuscular disease-related respiratory failure suffer from multiple afflictions that require the input of different specialists including pulmonologists, physiotherapists, occupational therapists, speech and language therapists, and dieticians [12]. This can result in frequent hospital appointments, which are made more difficult as these patients often have physical impairments requiring mobility assistance. Therefore, there is understandable interest in the use of telemedicine to reduce the burden of travel for these patients. Despite this, the literature to support the use of telemedicine in this group, particularly in adults, is limited.

The first trial to investigate the feasibility of home initiation of NIV in patients with either neuromuscular or chest wall disease demonstrated that it is as effective as hospital set-up in reducing $\mathrm{PaCO}_{2}$ and health-related QoL [92]. Similarly, another randomised study has recently demonstrated that home initiation with telemedicine support was noninferior to hospital set-up, with potential cost savings of up to EUR 3200 per patient [93]. A small cohort study demonstrated the feasibility of the delivery of a chest physiotherapy programme based on remote monitoring of pulse oximetry in patients with neuromuscular disease. This resulted in a reduction in hospitalisations and presentations to the emergency department [94]. Small case series have reported on the long-term management of adult patients with congenital muscular dystrophies, demonstrating the feasibility of this approach [95, 96]. Large-scale randomised studies are difficult to conduct in this patient group due to small numbers of patients being managed in individual centres and considerable heterogeneity within the patient populations [97]. The lack of comprehensive evidence, therefore, is likely to be a barrier to wider adoption of telemedicine in this group.

There are more supportive data for the use of telemedicine in the paediatric neuromuscular population. A case-control study compared telemonitoring (including planned weekly overnight recording of oxygen saturations, heart rate and ventilation) with usual care in young neuromuscular patients receiving HMV. They demonstrated that while telemonitoring did not reduce the frequency of exacerbations, it did result in fewer hospital admissions and hospital length of stay was shorter. They also demonstrated no detriment to caregiver burden and excellent caregiver satisfaction [98]. Cohort studies in children receiving HMV have reported that telemedicine via video consultations [99] and a remote ventilation monitoring system [100] was acceptable and appreciated by families and may have reduced the need for in-person reviews and emergency department presentations. In children receiving invasive mechanical ventilation at home supported by a telemedicine service, family satisfaction with the care provided was high. They reported that families felt telemedicine had reduced the need for hospital visits without any detriment to the child's QoL or safety [101]. Another study in tracheotomised children reported that the majority of life-threatening events suffered at home were managed successfully using telemedicine, preventing hospital admission [102]. A recent study has reported on the restrictions that SARS-CoV-2 has placed upon planned in-person reviews of paediatric HMV patients, and how they successfully managed most problems using remote technology [103].

\section{Opportunities and challenges in the use of telemedicine in chronic respiratory failure}

There is considerable potential for the expansion of telemedicine services that could be offered to patients with chronic respiratory failure. The validity and acceptability of remote pulmonary function tests performed by the patient and caregiver in patients with ALS has been demonstrated [104]. This would 
reduce the need for patients to attend hospital for routine monitoring, and with the wider availability of home-based initiation of NIV, the need for attendance at outpatient clinics in person may be significantly reduced. A toolset to conduct remote assessments of ALS patients, involving remote ventilator monitoring, digital speech analysis, remote cognitive assessment and audiovisual neurological assessment has been proposed to prevent hospital attendance during the SARS-CoV-2 pandemic [105]. If effective and financially viable, this model is likely to persist beyond the pandemic and extend to patient groups other than ALS, to reduce the burden of hospital attendance on these patients.

Recently, a report of a comprehensive telemonitoring system using $5 \mathrm{G}$ mobile data technology has demonstrated the rich amount of data that can be obtained from patients remotely [106]. With the distribution of 5G mobile technology, there is potential for larger amounts of rapid and reliable data transmission, which may contribute to its wider acceptance and uptake. PÉPIN et al. [107] note the potential of "big data capture" using telemedicine. Continuous recording of domiciliary ventilator data will result in huge repositories of data that can be interrogated to answer research questions, pose new hypotheses and facilitate personalised care of patients with chronic respiratory failure. International collaboration may also answer important questions about less common causes of chronic respiratory failure. This is particularly useful, because the feasibility of conducting large, randomised studies on telemedicine, particularly in neuromuscular diseases, is considered to be low [108].

The direct impact of the SARS-CoV-2 pandemic on the acceptance and use of telemedicine has not yet been formally evaluated, but given the rapid uptake of telemedicine services across the full spectrum of healthcare [109-111], it is likely that telemedicine is now here to stay. A survey of centres delivering domiciliary ventilator therapy has revealed a considerable reduction in in-patient assessment and treatment during the pandemic [112]. Although this did not result in a significant increase in telemedicine use (services were suspended during the pandemic), the authors conclude that there is now a need to use new telemedicine technologies to be able sustain services during the remainder of the pandemic and future pandemics. This drive will probably result in more research with stronger evidence either supporting or against telemedicine use in patients with chronic respiratory failure. This will be important as, prior to the SARS-CoV-2 pandemic, evidence suggested that telemedicine may not be widely accepted. A 2013 review of patients with chronic respiratory conditions reported low acceptance of telehealth technologies to deliver care [113]. A review of telemedicine in developing countries highlighted that the technological infrastructure in these countries may not be able to support the requirements of advanced telemedicine solutions that are being used in the developed world [114]. Finally, limited cellular phone use, particularly smartphone use, in the elderly may also provide a barrier to its widespread use. A survey of the elderly in New Zealand revealed low use of cellular phones and the internet in octogenarians [115], while the use of smartphones for medical support in a South Korean population was associated with younger age [116]. Encouragingly however, with time, smartphone use appears to be increasing substantially among the elderly in Western populations [117]. With further development, and the increased prioritisation of telemedicine due to the SARS-CoV-2 pandemic, these barriers are likely to diminish. Patients will become more accustomed to its use as routine practice, developing countries will clearly undergo technological development and with successive generations, cellular phone technologies will be more widely used.

Despite the advances made during the SARSCoV-2 pandemic, the available evidence base and significant logistical and legal challenges are likely to persist as barriers to the widespread roll-out of telemedicine (table 2). This review has demonstrated the broad spread of evidence currently available for telemedicine in chronic respiratory failure. Most evidence syntheses have been inconclusive due to the small number of studies, as well as inclusion of different patient groups, different control groups and the assessment of different outcome measures. Without standardisation of study design and the development of a core outcome set in this field, it will remain difficult to make conclusive judgements. The heterogeneous nature of the patients included in studies to date make it difficult to identify which patient group will benefit from telemedicine [118]. Patients of mixed severity, and at times, mixed disease groups, are included in these studies, which is likely to contribute to the mixed results obtained. Furthermore, different telemedicine platforms and technologies are assessed in different studies, again presenting difficulty in identifying the approaches that would be of most benefit in this group.

Following the end of the SARS-CoV-2 pandemic, the legal issues that remain outstanding will need to be addressed. The public interest argument may have allowed telemedicine to be used where formal data sharing agreements were not in place and extensive assessment of data security was not performed [119, 120]. Following the end of the pandemic, these issues will likely be reviewed and the concerns about data sharing and confidentiality of personal data during electronic transmission will need to be addressed [121]. Prior to the pandemic, a survey of healthcare professionals and medical students demonstrated concern about data security, responsibility for data ownership, the patient-doctor interpersonal relationship and reliability of electronically transmitted information [122]. These concerns will need to be addressed and reassurance provided to healthcare professionals, patients and regulatory bodies before telemedicine can be widely disseminated. 
Telemedicine and management of chronic respiratory failure

Table 2 Opportunities and challenges in the introduction of telemedicine services in the management of patients with chronic respiratory failure

\begin{tabular}{ll}
\hline Opportunities & Challenges \\
\hline $\begin{array}{l}\text { Reduce travel time and frequency of attendance at } \\
\text { outpatient clinics [44, 82] }\end{array}$ & $\begin{array}{l}\text { Patient and healthcare professional acceptance of } \\
\text { telemedicine }[74,122]\end{array}$ \\
$\begin{array}{l}\text { More frequent monitoring and contact with patients [48, 49] } \\
\text { Feduce caregiver burden [101] }\end{array}$ & $\begin{array}{l}\text { Infrastructure costs [123] } \\
\text { Data security and reliability [119, 121, 122] } \\
\text { Improve QoL [40, 47, 92] }\end{array}$ \\
$\begin{array}{l}\text { Reduce attendance at primary care and emergency } \\
\text { department [37, 38, 63, 94] }\end{array}$ & An increase in workload to monitor large amounts of data [123] \\
$\begin{array}{l}\text { Reduce hospitalisations [36, 38, 63, 64, 79, 94] } \\
\text { Increase patient empowerment with immediate feedback [69] }\end{array}$ & \\
$\begin{array}{l}\text { Potential for long-term cost savings [46, 87] } \\
\text { Opportunity for big data analysis to answer outstanding } \\
\text { questions in rare conditions [107] }\end{array}$ & \\
\hline
\end{tabular}

To support the continued provision of telemedicine programmes, much more detailed analysis of the organisational costs to the healthcare system is required. While the potential for reducing healthcare costs by reducing outpatient visits and hospitalisations is attractive, this needs to be evaluated against the infrastructure costs required to provide a telemedicine platform, as well as the potential cost of increased and more frequent interactions requiring more clinical staff to deliver this [123]. Healthcare systems will also need to develop stringent renumeration policies to ensure all patient encounters are captured and accounted for. These changes may result in an increase in cost.

\section{Summary}

Patients with chronic respiratory failure and their caregivers can experience significant difficulty through the requirement to attend multiple in-hospital appointments and in managing their care using complex devices at home. Telemedicine is an opportunity to reduce this burden on the patient and caregiver by facilitating closer and more frequent contact with the healthcare team. Using telephone and cloud-based systems, the healthcare team is able to remotely monitor vital signs and ventilator data and make adjustments accordingly. The existing evidence for the use of telemedicine in chronic respiratory failure to improve clinical outcomes is equivocal. Although the SARSCoV-2 pandemic has presented an opportunity to increase the pace of its introduction, significant challenges need to be addressed before it will be widely adopted. Stronger evidence supporting its use is needed, particularly around which patient groups will benefit the most. Challenges around secure data transmission, acceptability to patients and healthcare professionals, and financial models remain. The end of the pandemic may act as the catalyst to deliver studies that investigate these outstanding issues and ultimately provide conclusive evidence.

\section{Key points}

- There is ample scope to improve the delivery of care for patients with chronic respiratory failure with the adoption of telemedicine systems.

- Telemedicine may help to reduce the requirement for acute care in these vulnerable patients, improve QoL and potentially result in financial savings to the healthcare system. The evidence base to support the adoption of telemedicine in chronic respiratory failure remains equivocal.

- Challenges that need to be addressed include identifying the target population that will benefit from telemedicine, understanding the costeffectiveness of these interventions and legal considerations such as data security and clinician responsibility. 


\section{Self-evaluation questions}

1. Which of the following healthcare professional roles can be delivered by telemedicine?
a) Consultation
b) Diagnostic assessments
c) Initiation of treatment
d) Long-term monitoring of patient

2. Which of the following are not necessary components of the infrastructure of a telemedicine service set-up?

a) Central server

b) $5 G$-enabled devices

c) Internet connection at each location

d) Centralised coordinator

3. Which of the following is not an outstanding challenge that needs to be considered when developing a telemedicine programme?

a) Healthcare professional and patient familiarity with technology required

b) Feasibility of the delivery of a telemedicine programme for patients with chronic respiratory failure

c) Finance

d) Data security

\section{Affiliations}

\section{Neeraj M. Shah ${ }^{1,2,3}$, Georgios Kaltsakas ${ }^{1,2,3}$}

'Lane Fox Respiratory Service, St Thomas' Hospital, Guy's and St Thomas' NHS Foundation Trust, London, UK. 'Lane Fox Clinical Respiratory Physiology Centre, Guy's and St Thomas' NHS Foundation Trust, London, UK.

${ }^{3}$ Centre for Human and Applied Physiological Sciences (CHAPS), King's College London, London, UK.

\section{Conflict of interest}

N.M. Shah has nothing to disclose. G. Kaltsakas has nothing to disclose.

\section{References}

1. Ekeland AG, Bowes A, Flottorp S. Effectiveness of telemedicine: a systematic review of reviews. Int J Med Inform 2010; 79: 736-771.

2. Flodgren $\mathrm{G}$, Rachas A, Farmer AJ, et al. Interactive telemedicine: effects on professional practice and health care outcomes. Cochrane Database Syst Rev 2015; 2015: CD002098.

3. Jayakody A, Bryant J, Carey M, et al. Effectiveness of interventions utilising telephone follow up in reducing hospital readmission within 30 days for individuals with chronic disease: a systematic review. BMC Health Serv Res 2016; 16: 403.

4. Eze ND, Mateus C, Cravo Oliveira Hashiguchi T. Telemedicine in the OECD: an umbrella review of clinical and costeffectiveness, patient experience and implementation PLoS One 2020; 15: e0237585.

5. Gregersen TL, Green A, Frausing E, et al. Do telemedical interventions improve quality of life in patients with COPD? A systematic review. Int J Chron Obstruct Pulmon Dis 2016; 11: 809-822

6. Latifi R, Doarn CR. Perspective on COVID-19: finally, telemedicine at center stage. Telemed J E Health 2020; 26 : 1106-1109.

7. Hardinge M, Annandale J, Bourne S, et al. British Thoracic Society guidelines for home oxygen use in adults: accredited by NICE. Thorax 2015; 70: Suppl. 1, i1-i43.
8. Ergan B, Oczkowski S, Rochwerg B, et al. European Respiratory Society guidelines on long-term home noninvasive ventilation for management of COPD. Eur Respir J 2019; 54: 1901003.

9. Simonds AK. Home mechanical ventilation: an overview. Ann Am Thorac Soc 2016; 13: 2035-2044.

10. Meservey AJ, Burton MC, Priest J, et al. Risk of readmission and mortality following hospitalization with hypercapnic respiratory failure. Lung 2020; 198: 121-134.

11. Crockett AJ, Moss JR, Cranston JM, et al. The effects of home oxygen therapy on hospital admission rates in chronic obstructive airways disease. Monaldi Arch Chest Dis 1993; 48: 445-446.

12. Shah NM, Murphy PB, Kaltsakas G. The adult multidisciplinary respiratory neuromuscular clinic. Breathe 2020; 16: 200121.

13. Schellenberg KL, Hansen G. Patient perspectives on transitioning to amyotrophic lateral sclerosis multidisciplinary clinics. J Multidiscip Healthc 2018; 11: 519-524.

14. Marin JM, Soriano JB, Carrizo SJ, et al. Outcomes in patients with chronic obstructive pulmonary disease and obstructive sleep apnea: the overlap syndrome. Am J Respir Crit Care Med 2010; 182: 325-331.

15. Long-Term Oxygen Treatment Trial Research Group, Albert $\mathrm{RK}, \mathrm{Au} \mathrm{DH}$, et al. A randomized trial of long-term oxygen for COPD with moderate desaturation. N Engl J Med 2016; 375: $1617-1627$. 
16. Yüksel A, Çiftçi F, Çiledağ A, et al. The effects of home noninvasive ventilation on the quality of life and physiological parameters of patients with chronic respiratory failure. Clin Respir J 2020; 14: 880-888.

17. Piepers S, van den Berg JP, Kalmijn S, et al. Effect of noninvasive ventilation on survival, quality of life, respiratory function and cognition: a review of the literature. Amyotroph Lateral Scler 2006; 7: 195-200.

18. Eaton T, Lewis C, Young P, et al. Long-term oxygen therapy improves health-related quality of life. Respir Med 2004; 98 : 285-293.

19. Boussaïd G, Stalens C, Devaux C, et al. Impact of mechanical ventilation methods on the life perception of subjects with Duchenne muscular dystrophy: French cross-sectional survey. Respir Care 2020; 65: 1712-1720.

20. Tissot A, Jaffre $S$, Gagnadoux F, et al. Home non-invasive ventilation fails to improve quality of life in the elderly: results from a Multicenter Cohort Study. PLoS One 2015; 10: e0141156.

21. Okubadejo AA, Paul EA, Jones PW, et al. Does long-term oxygen therapy affect quality of life in patients with chronic obstructive pulmonary disease and severe hypoxaemia? Eur Respir J 1996; 9: 2335-2339.

22. Nonoyama ML, Brooks D, Guyatt GH, et al. Effect of oxygen on health quality of life in patients with chronic obstructive pulmonary disease with transient exertional hypoxemia. Am J Respir Crit Care Med 2007; 176: 343-349.

23. Huttmann SE, Windisch W, Storre JH. Invasive home mechanical ventilation: living conditions and health-related quality of life. Respiration 2015; 89: 312-321.

24. Huttmann SE, Magnet FS, Karagiannidis C, et al. Quality of life and life satisfaction are severely impaired in patients with long-term invasive ventilation following ICU treatment and unsuccessful weaning. Ann Intensive Care 2018; 8: 38

25. Crimi C, Pierucci P, Carlucci A, et al. Long-term ventilation in neuromuscular patients: review of concerns, beliefs, and ethical dilemmas. Respiration 2019; 97: 185-196.

26. Findeis A, Larson JL, Gallo A, et al. Caring for individuals using home ventilators: an appraisal by family caregivers. Rehabil Nurs 1994; 19: 6-11.

27. Dale CM, King J, Nonoyama M, et al. Transitions to home mechanical ventilation. The experiences of Canadian ventilator-assisted adults and their family caregivers. Ann Am Thorac Soc 2018; 15: 357-364.

28. Ambrosino N, Vitacca M, Dreher M, et al. Tele-monitoring of ventilator-dependent patients: a European Respiratory Society Statement. Eur Respir J 2016; 48: 648-663.

29. Amar-Dolan LG, Horn MH, O'Connell B, et al. "This is how hard it is": family experience of Hospital-to-Home transition with a tracheostomy. Ann Am Thorac Soc 2020; 17: 860-868.

30. Soo Cl, Chan Y, Loh EC, et al. Telehealth: "virtual" lifeline for home-ventilated patients during the COVID-19 pandemic. ERJ Open Res 2020; 6: 00399-2020.

31. Hernandez C, Mallow J, Narsavage GL. Delivering telemedicine interventions in chronic respiratory disease. Breathe 2014; 10: 198-212.

32. Aliverti A. Wearable technology: role in respiratory health and disease. Breathe 2017; 13: e27-e36.

33. Hosseiniravandi $M$, Kahlaee $\mathrm{AH}$, Karim $\mathrm{H}$, et al. Home-based telerehabilitation software systems for remote supervising: a systematic review. Int J Technol Assess Health Care 2020; 36: 113-125.

34. Al Rajeh A, Steiner MC, Aldabayan Y, et al. Use, utility and methods of telehealth for patients with COPD in England and Wales: a healthcare provider survey. BMJ Open Respir Res 2019; 6: e000345.

35. Alrajeh AM, Aldabayan YS, Aldhair AM, et al. Global use, utility, and methods of tele-health in COPD: a health care provider survey. Int J Chron Obstruct Pulmon Dis 2019; 14: 1713-1719.

36. Lyth J, Lind L, Persson HL, et al. Can a telemonitoring system lead to decreased hospitalization in elderly patients? J Telemed Telecare 2019; 27: 46-53.

37. Segrelles Calvo G, Gómez-Suárez C, Soriano JB, et al. A home telehealth program for patients with severe COPD: the PROMETE study. Respir Med 2014; 108: 453-462.
38. Vitacca M, Bianchi L, Guerra A, et al. Tele-assistance in chronic respiratory failure patients: a randomised clinical trial. Eur Respir J 2009; 33: 411-418.

39. Walker PP, Pompilio PP, Zanaboni P, et al. Telemonitoring in chronic obstructive pulmonary disease (CHROMED). A randomized clinical trial. Am J Respir Crit Care Med 2018; 198: 620-628

40. Burioka N, Yamamoto A, Morimoto M, et al. Health-related quality of life in patients on home oxygen therapy with telemonitoring. Yonago Acta Med 2020; 63: 132-134.

41. Faria I, Gaspar C, Zamith M, et al. TELEMOLD project: oximetry and exercise telemonitoring to improve long-term oxygen therapy. Telemed J E Health 2014; 20: 626-632.

42. Vitacca M, Comini L, Tabaglio E, et al. Tele-assisted palliative homecare for advanced chronic obstructive pulmonary disease: a feasibility study. J Palliat Med 2019; 22: 173-178.

43. Sul AR, Lyu DH, Park DA. Effectiveness of telemonitoring versus usual care for chronic obstructive pulmonary disease: a systematic review and meta-analysis. J Telemed Telecare 2020; 26: 189-199.

44. Logie K, Welsh L, Ranganathan SC. Telehealth spirometry for children with cystic fibrosis. Arch Dis Child 2020; 105: 1203.

45. Conway S, Balfour-Lynn IM, De Rijcke K, et al. European Cystic Fibrosis Society standards of care: framework for the cystic fibrosis centre. J Cyst Fibros 2014; 13: S3-S22.

46. Tagliente I, Trieste L, Solvoll T, et al. Telemonitoring in Cystic Fibrosis: a 4-year assessment and simulation for the next 6 years. Interact J Med Res 2016; 5: e11.

47. Grzincich G, Gagliardini R, Bossi A, et al. Evaluation of a home telemonitoring service for adult patients with cystic fibrosis: a pilot study. J Telemed Telecare 2010; 16: 359-362.

48. Wood J, Mulrennan S, Hill K, et al. Telehealth clinics increase access to care for adults with cystic fibrosis living in rural and remote Western Australia. J Telemed Telecare 2016; 23: 673-679.

49. Bella S, Murgia F, Tozzi AE, et al. Five years of telemedicine in cystic fibrosis disease. Clin Ter 2009; 160: 457-460.

50. De Biase RV, Cristiani L, Paglia C, et al. Clinical and microbiological monitoring of Cystic Fibrosis patients, three years of follow-up via Tele-Medicine: an empirical research. Clin Ter 2020; 171: e381-e384.

51. Wilkinson OM, Duncan-Skingle F, Pryor JA, et al. A feasibility study of home telemedicine for patients with cystic fibrosis awaiting transplantation. J Telemed Telecare 2008; 14: 182-185.

52. Cox NS, Alison JA, Rasekaba T, et al. Telehealth in cystic fibrosis: a systematic review. J Telemed Telecare 2012; 18: 72-78

53. Ketchell RI. Telemedicine is the way forward for the management of cystic fibrosis - the case in favour. Paediatr Respir Rev 2018; 26: 19-21.

54. Compton M, Soper M, Reilly B, et al. A feasibility study of urgent implementation of cystic fibrosis multidisciplinary telemedicine clinic in the face of COVID-19 pandemic: single-center experience. Telemed J E Health 2020; 26: 978-984.

55. Moor CC, Wapenaar M, Miedema JR, et al. A home monitoring program including real-time wireless home spirometry in idiopathic pulmonary fibrosis: a pilot study on experiences and barriers. Respir Res 2018; 19: 105.

56. Moor CC, Mostard RLM, Grutters JC, et al. Home monitoring in patients with idiopathic pulmonary fibrosis. a randomized controlled trial. Am J Respir Crit Care Med 2020; 202: 393-401.

57. Yuen HK, Lowman JD, Oster RA, et al. Home-based pulmonary rehabilitation for patients with idiopathic pulmonary fibrosis: a pilot study. J Cardiopulm Rehabil Prev 2019; 39: 281-284.

58. Antoniou KM, Raghu G, Tzilas V, et al. Management of patients with interstitial lung disease in the midst of the COVID-19 pandemic. Respiration 2020; 99: 625-627.

59. Bryant MS, Bandi VD, Nguyen CK, et al. Telehealth pulmonary rehabilitation for patients with severe chronic obstructive pulmonary disease. Fed Pract 2019; 36: 430-435.

60. Selzler AM, Wald J, Sedeno M, et al. Telehealth pulmonary rehabilitation: a review of the literature and an example

\section{Suggested answers}

1. $a-d$.

2. b.

3. b. 
of a nationwide initiative to improve the accessibility of pulmonary rehabilitation. Chron Respir Dis 2018; 15: 41-47.

61. Almojaibel AA, Munk N, Goodfellow LT, et al. Health care practitioners' determinants of telerehabilitation acceptance. Int J Telerehabil 2020; 12: 43-50.

62. Wootton SL, King M, Alison JA, et al. COVID-19 rehabilitation delivered via a telehealth pulmonary rehabilitation model: a case series. Respirol Case Rep 2020; 8: e00669.

63. Vasilopoulou M, Papaioannou Al, Kaltsakas G, et al. Home-based maintenance tele-rehabilitation reduces the risk for acute exacerbations of COPD, hospitalisations and emergency department visits. Eur Respir J 2017; 49: 1602129 .

64. Bhatt SP, Patel SB, Anderson EM, et al. Video telehealth pulmonary rehabilitation intervention in chronic obstructive pulmonary disease reduces 30-day readmissions. Am J Respir Crit Care Med 2019; 200: 511-513.

65. Godtfredsen N, Frølich A, Bieler T, et al. 12-months follow-up of pulmonary tele-rehabilitation versus standard pulmonary rehabilitation: a multicentre randomised clinical trial in patients with severe COPD. Respir Med 2020; 172: 106129.

66. Hansen $\mathrm{H}$, Bieler $\mathrm{T}$, Beyer $\mathrm{N}$, et al. Supervised pulmonary tele-rehabilitation versus pulmonary rehabilitation in severe COPD: a randomised multicentre trial. Thorax 2020; 75 413-421.

67. Chan C, Inskip JA, Kirkham AR, et al. A smartphone oximeter with a fingertip probe for use during exercise training: usability, validity and reliability in individuals with chronic lung disease and healthy controls. Physiotherapy 2019; 105: 297-306.

68. Loeckx M, Rabinovich RA, Demeyer H, et al. Smartphonebased physical activity telecoaching in chronic obstructive pulmonary disease: mixed-methods study on patient experiences and lessons for implementation. JMIR Mhealth Uhealth 2018; 6: e200.

69. Inskip JA, Lauscher HN, Li LC, et al. Patient and health care professional perspectives on using telehealth to deliver pulmonary rehabilitation. Chron Respir Dis 2018; 15: 71-80.

70. Bonnevie T, Smondack P, Elkins M, et al. Advanced telehealth technology improves home-based exercise therapy for people with stable chronic obstructive pulmonary disease: a systematic review. J Physiother 2021; 67: 27-40

71. Cox NS, Dal Corso S, Hansen H, et al. Telerehabilitation for chronic respiratory disease. Cochrane Database Syst Rev 2021; 1: CD013040.

72. Jácome C, Marques A, Oliveira A, et al. Pulmonary telerehabilitation: an international call for action. Pulmonology 2020; 26: 335-337.

73. Houchen-Wolloff L, Steiner MC. Pulmonary rehabilitation at a time of social distancing: prime time for tele-rehabilitation? Thorax 2020; 75: 446-447.

74. Masefield S, Vitacca M, Dreher M, et al. Attitudes and preferences of home mechanical ventilation users from four European countries: an ERS/ELF survey. ERJ Open Res 2017; 3: 00015-2017.

75. Crimi C, Noto A, Princi P, et al. Domiciliary non-invasive ventilation in COPD: an international survey of indications and practices. COPD 2016; 13: 483-490.

76. Duiverman ML, Vonk JM, Bladder G, et al. Home initiation of chronic non-invasive ventilation in COPD patients with chronic hypercapnic respiratory failure: a randomised controlled trial. Thorax 2020; 75: 244-252.

77. Bertini S, Picariello M, Gorini M, et al. Telemonitoring in chronic ventilatory failure: a new model of survellaince, a pilot study. Monaldi Arch Chest Dis 2012; 77: 57-66.

78. Borel JC, Pelletier J, Taleux N, et al. Parameters recorded by software of non-invasive ventilators predict COPD exacerbation: a proof-of-concept study. Thorax 2015; 70: 284-285.

79. Vitacca M, Paneroni M, Grossetti F, et al. Is there any additional effect of tele-assistance on long-term care programmes in hypercapnic COPD patients? A retrospective study. COPD 2016; 13: 576-582.

80. Chatwin M, Hawkins G, Panicchia L, et al. Randomised crossover trial of telemonitoring in chronic respiratory patients (TeleCRAFT trial). Thorax 2016; 71: 305-311.
81. de Almeida JP, Pinto AC, Pereira J, et al. Implementation of a wireless device for real-time telemedical assistance of home-ventilated amyotrophic lateral sclerosis patients: a feasibility study. Telemed J E Health 2010; 16: 883-888.

82. Scalvini S, Bernocchi P, Zanelli E, et al. Maugeri Centre for Telehealth and Telecare: a real-life integrated experience in chronic patients. J Telemed Telecare 2018; 24: 500-507.

83. Pinto A, Almeida JP, Pinto S, et al. Home telemonitoring of non-invasive ventilation decreases healthcare utilisation in a prospective controlled trial of patients with amyotrophic lateral sclerosis. J Neurol Neurosurg Psychiatry 2010; 81: 1238-1242.

84. Selkirk SM, Washington MO, McClellan F, et al. Delivering tertiary centre specialty care to ALS patients via telemedicine: a retrospective cohort analysis. Amyotroph Lateral Scler Frontotemporal Degener 2017; 18: 324-332.

85. Ando $\mathrm{H}$, Ashcroft-Kelso $\mathrm{H}$, Halhead $\mathrm{R}$, et al. Experience of telehealth in people with motor neurone disease using noninvasive ventilation. Disabil Rehabil Assist Technol 2019; in press [https://doi. org/10.1080/17483107.2019.1659864].

86. Lopes de Almeida JP, Pinto A, Pinto S, et al. Economic cost of home-telemonitoring care for BiPAP-assisted ALS individuals. Amyotroph Lateral Scler 2012; 13: 533-537.

87. Paganoni S, van de Rijn M, Drake K, et al. Adjusted cost analysis of video televisits for the care of people with amyotrophic lateral sclerosis. Muscle Nerve 2019; 60: 147-154.

88. Vitacca M, Paneroni M, Trainini D, et al. At home and on demand mechanical cough assistance program for patients with amyotrophic lateral sclerosis. Am J Phys Med Rehabil 2010; 89: 401-406

89. Helleman J, Kruitwagen ET, van den Berg LH. The current use of telehealth in ALS care and the barriers to and facilitators of implementation: a systematic review. Amyotroph Lateral Scler Frontotemporal Degener 2020; 21: 167-182.

90. Haulman A, Geronimo A, Chahwala A, et al. The use of telehealth to enhance care in ALS and other neuromuscular disorders. Muscle Nerve 2020; 61: 682-691.

91. Hobson EV, Baird WO, Cooper CL, et al. Using technology to improve access to specialist care in amyotrophic lateral sclerosis: a systematic review. Amyotroph Lateral Scler Frontotemporal Degener 2016; 17: 313-324.

92. Hazenberg A, Kerstjens HA, Prins SC, et al. Initiation of home mechanical ventilation at home: a randomised controlled trial of efficacy, feasibility and costs. Respir Med 2014; 108 : 1387-1395

93. van den Biggelaar RJM, Hazenberg A, Cobben NAM, et al. A randomized trial of initiation of chronic noninvasive mechanical ventilation at home vs in-hospital in patients with neuromuscular disease and thoracic cage disorder: The Dutch Homerun Trial. Chest 2020; 158: 2493-2501.

94. Garuti G, Bagatti S, Verucchi E, et al. Pulmonary rehabilitation at home guided by telemonitoring and access to healthcare facilities for respiratory complications in patients with neuromuscular disease. Eur J Phys Rehabil Med 2013; 49: 51-57.

95. Portaro S, Calabro RS, Bramanti P, et al. Telemedicine for facioscapulo-humeral muscular dystrophy: a multidisciplinary approach to improve quality of life and reduce hospitalization rate? Disabil Health J 2018; 11: 306-309.

96. Zamarrón C, Morete E, González F. Telemedicine system for the care of patients with neuromuscular disease and chronic respiratory failure. Arch Med Sci 2014; 10: 1047-1051.

97. Bach JR, Chiarello G, Weiss W, et al. Is there value in using randomized placebo controlled trials in neuromuscular disease? Expert Rev Neurother 2021; 21: 5-7.

98. Trucco F, Pedemonte M, Racca F, et al. Tele-monitoring in paediatric and young home-ventilated neuromuscular patients: a multicentre case-control trial. J Telemed Telecare 2019; 25: 414-424.

99. Casavant DW, McManus ML, Parsons SK, et al. Trial of telemedicine for patients on home ventilator support: feasibility, confidence in clinical management and use in medical decision-making. J Telemed Telecare 2014; 20: 441-449. 
100. Zhou J, Liu DB, Zhong JW, et al. Feasibility of a remote monitoring system for home-based non-invasive positive pressure ventilation of children and infants. Int J Pediatr Otorhinolaryngol 2012; 76: 1737-1740.

101. Muñoz-Bonet JI, López-Prats JL, Flor-Macián EM, et al. Usefulness of telemedicine for home ventilator-dependent children. J Telemed Telecare 2020; 26: 207-215.

102. Muñoz-Bonet JI, López-Prats JL, Flor-Macián EM, et al. Medical complications in a telemedicine home care programme for paediatric ventilated patients. J Telemed Telecare 2019; 26: 462-473.

103. Onofri A, Pavone M, De Santis S, et al. Telemedicine in children with medical complexity on home ventilation during the COVID-19 pandemic. Pediatric Pulmonol 2021; in press [https://doi.org/10.1002/ppul.25289].

104. Geronimo A, Simmons Z. Evaluation of remote pulmonary function testing in motor neuron disease. Amyotroph Lateral Scler Frontotemporal Degener 2019; 20: 348-355.

105. Bombaci A, Abbadessa G, Trojsi F, et al. Telemedicine for management of patients with amyotrophic lateral sclerosis through COVID-19 tail. Neurol Sci 2021; 42: 9-13.

106. Angelucci A, Kuller D, Aliverti A. A home telemedicine system for continuous respiratory monitoring. IEEE J Biomed Health Inform 2020; in press [https://doi.org/10.1109/ JBHI.2020.3012621].

107. Pépin JL, Tamisier R, Hwang D, et al. Does remote monitoring change OSA management and CPAP adherence? Respirology 2017; 22: 1508-1517.

108. Hobson EV, Baird WO, Bradburn M, et al. Using telehealth in motor neuron disease to increase access to specialist multidisciplinary care: a UK-based pilot and feasibility study. BMJ Open 2019; 9: e028525.

109. Monaghesh E, Hajizadeh A. The role of telehealth during COVID-19 outbreak: a systematic review based on current evidence. BMC Public Health 2020; 20: 1193

110. Webster P. Virtual health care in the era of COVID-19. Lancet 2020; 395: 1180-1181.

111. Hollander JE, Carr BG. Virtually perfect? Telemedicine for Covid-19. N Engl J Med 2020; 382: 1679-1681.

112. Grote L, McNicholas WT, Hedner J, et al. Sleep apnoea management in Europe during the COVID-19 pandemic: data from the European Sleep Apnoea Database (ESADA) Eur Respir J 2020; 55: 2001323.
113. Duplaga $M$. The acceptance of e-health solutions among patients with chronic respiratory conditions. Telemed J $E$ Health 2013; 19: 683-691.

114. Combi C, Pozzani G, Pozzi G. Telemedicine for developing countries. A survey and some design issues. App/ Clin Inform. 2016; 7: 1025-1050.

115. Atlas A, Muru-Lanning M, Moyes S, et al. Cell phone and technology use by octogenarians. J Prim Health Care 2020; 12: 35-40.

116. Oh YS, Choi EY, Kim YS. Predictors of smartphone uses for health information seeking in the Korean elderly. Soc Work Public Health 2018; 33: 43-54.

117. Pew Research Center. Tech adoptions climbs among older adults. www.pewresearch.org/internet/2017/05/17/ technology-use-among-seniors/ Date last updated: 17 May 2017.

118. Vitacca M. Telemonitoring in patients with chronic respiratory insufficiency: expectations deluded? Thorax 2016; 71: 299

119. Vidal-Alaball J, Acosta-Roja R, Pastor Hernández N, et al. Telemedicine in the face of the COVID-19 pandemic. Aten Primaria 2020; 52: 418-422.

120. Mehrotra A, Nimgaonkar A, Richman B. Telemedicine and medical licensure - potential paths for reform. NEnglJ Med 2021; 384: 687-690

121. Nittari G, Khuman R, Baldoni S, et al. Telemedicine practice: review of the current ethical and legal challenges. Telemed J E Health 2020; 26: 1427-1437.

122. Wernhart A, Gahbauer S, Haluza D. eHealth and telemedicine: Practices and beliefs among healthcare professionals and medical students at a medical university. PLoS One 2019; 14: e0213067

123. Michaud TL, Zhou J, McCarthy MA, et al. Costs of home-based telemediicine programs: a systematic review. Int J Technol Assess Health Care 2018; 34: 410-418.

124. Kyriacou E, Pavlopoulos S, Berler A, et al. Multipurpose HealthCare Telemedicine Systems with mobile communication link support. Biomed Eng Online 2003; 2: 7.

125. Angelucci $A$, Aliverti $A$. Telemonitoring systems for respiratory patients: technological aspects. Pulmonology 2020; 26: 221-232.

126. Abo-Zahhad M, Ahmed SM, Elnahas O. A wireless emergency telemedicine system for patients monitoring and diagnosis. Int J Telemed Appl 2014; 2014: 380787. 